\title{
MODELO DE CÁLCULO ANALÍTICO DEL TIEMPO REQUERIDO PARA QUE UNA CORRIENTE DE ARCO DC QUE SIMULA UN RAYO FUNDA UN PORCENTAJE DE LA SECCIÓN DE UN CABLE DE GUARDA DE LÍNEAS DE TRANSMISIÓN
}

\begin{abstract}
Juan C. Escobar ${ }^{1}$, Esteban Velilla, Germán Moreno²
${ }^{1}$ Ingeniero electricista, Universidad de Antioquia, Grupo de Investigación GIMEL, Medellín-Colombia, jcescobaralvarez@gmail.com

${ }^{2}$ Magíster en ingeniería, Universidad de Antioquia, Grupo de Investigación GIMEL, Medellín-Colombia esteban.velilla@udea.edu.co

RESUMEN

Se desarrolló un modelo de cálculo analítico del tiempo requerido para que una determinada corriente de arco DC que simula un rayo funda un porcentaje de la sección de un cable de guarda de líneas de transmisión con base en la energía transferida al cable de guarda cuando este es impactado por un rayo. En el cálculo de la energía transferida al cable de guarda cuando es impactado por un rayo se tiene en cuenta la energía por emisión o absorción de electrones cuando se produce el arco de plasma y se desprecian el efecto Joule y la transferencia de calor por radiación y convección. El modelo desarrollado se calibró con los resultados reportados por Mikimasa Iwata y se encontró que el modelo desarrollado se ajusta bien.
\end{abstract}

Palabras clave: Cable de guarda, cable OPGW, rayo, descarga eléctrica atmosférica, daño, arco de plasma, líneas de transmisión.

Recibido: 7 de marzo de 2018. Aceptado: 24 de septiembre de 2018

Received: March 7th, 2018. Accepted: September 24th, 2018

\section{ANALYTICAL MODEL TO CALCULATE THE REQUIRED TIME OF A DC CURRENT SIMULATING A LIGHTNING STRIKE TO MELTS A PERCENTAGE OF THE SECTION OF A TRANSMISSION POWER LINE SHIELD WIRE}

\begin{abstract}
An analytical model for calculating the time required for a DC current simulating the current of a lightning melts a certain percentage of a transmission power line shield wire section, based on the energy transfered to the shield wire by a lightning strike. In the calculation of the energy transfered to the shield wire by a lightning strike when the plasma arcjet is produced, the energy due to electrons emission or absortion for the shield wire is taken in to account and the energy due to joule effect and convection and radiation heat are neglected. The developed model was adjusted with results presented by Mikimasa Iwata and it was found that the developed model is well adjusted.
\end{abstract}

Keywords: Shield wire, OPGW cable, lightning, lightning strike, damage, plasma arcjet, overhead power lines.

Cómo citar este artículo: J. C. Escobar, E. Velilla, G. Moreno, "Modelo de cálculo analítico del tiempo requerido para que una corriente de arco de que simula un rayo funda un porcentaje de la sección de un cable de guarda de líneas de transmisión", Revista Politécnica, vol. 14, no. 27 pp.39-44, 2018. https://doi.org/10.33571/rpolitec.v14n27a4 


\section{INTRODUCCIÓN}

Las líneas de transmisión se componen principalmente de torres, puestas a tierra, aisladores y cables. Los cables se dividen en: 1) conductores de fase utilizados para transportar la energía eléctrica y diseñados para este fin, y 2) cables de guarda que son diseñados para proteger a los conductores de fase de descargas eléctricas atmosféricas (impactos directos por rayos), y llevar a tierra las corrientes no deseadas (generadas por rayos, corto circuitos 0 por inducciones). Actualmente, el cable de guarda también es utilizado para la transmisión de datos de comunicación a través de la fibra óptica inmersa en dichos conductores, denominados OPGW, de las siglas en inglés Optical Power Ground Wire [1].

Las líneas de transmisión (LT) son las encargadas de transportar la energía eléctrica de las centrales de generación a los centros de consumo; por lo cual cruzan distancias considerables para cumplir con dicho objetivo, es posible que a lo largo de su trayecto se encuentren con diversidad de microclimas que involucren variedad de temperaturas, radiaciones solares, velocidades y direcciones de vientos, topografías, resistividad de terreno, nivel ceráunico, entre otras, y puedan así, afectar tanto el desempeño de los conductores como la calidad y continuidad del servicio eléctrico ofrecido por las empresas de transmisión, si estos conductores no están diseñados adecuadamente para las condiciones específicas a las que se han de enfrentar.

Colombia es un país con un alto nivel de descargas atmosféricas [2] y se han presentado casos en los cuales las descargas eléctricas atmosféricas han provocado rompimiento de cables de guarda, provocando interrupciones del suministro de energía eléctrica lo cual causa sobrecostos para las empresas de transmisión de energía eléctrica, razón por la cual es necesario estudiar la manera de calcular el daño que un rayo causa a los cables de guarda de las líneas de transmisión.

En [3] presentan los conceptos con los que desarrollaron un método para calcular el tiempo requerido para que una corriente de arco DC que simula un rayo funda un porcentaje de la sección de un cable de guarda de líneas de transmisión, sin embargo no presentan el modelo de cálculo, por lo tanto el objetivo de este artículo es desarrollar un modelo de cálculo y comparar los resultados que se obtienen con el modelo desarrollado con los resultados presentados en [3].

\section{METODOLOGÍA}

En [3] no se describe con detalle la manera de calcular el tiempo requerido para fundir un porcentaje del cable OPGW. Teniendo en cuenta los conceptos indicados en [3] se desarrolló un modelo para calcular el tiempo requerido para fundir un porcentaje de la sección del cable de guarda. A continuación se muestran los conceptos presentados en [3]:

\subsection{CONCEPTOS PRESENTADOS EN [3]}

\subsubsection{ENERGIA NECESARIA PARA FUNDIR LOS HILOS DE CABLE OPGW}

Según [3] la energía requerida para fundir los hilos del cable es la suma de la energía requerida para incrementar la temperatura del cable desde la temperatura ambiente hasta la temperatura de fusión y la energía requerida para lograr la fusión después de alcanzar la temperatura de fusión. La primera energía se calcula usando el calor específico y la densidad del material del cable. El calor específico es una propiedad del material, esta propiedad es la energía necesaria para elevar un grado de temperatura una unidad de masa de dicho material. Aunque el calor específico varía con la temperatura, en los cálculos se asume un valor promedio entre el valor a la temperatura ambiente y el valor a la temperatura de fusión. La segunda energía se calcula con el concepto de calor latente de fusión y la masa del material del cable. El calor latente de fusión es el calor necesario para que una determinada cantidad de un material se funda después de haber alcanzado la temperatura de fusión.

\subsubsection{FORMAS DE TRANSFERENCIA DE CALOR DEL ARCO DE PLASMA AL CABLE DE GUARDA}

En [3] se presentó una ecuación que tiene en cuenta los diferentes fenómenos que transfieren energía al cable cuando es impactado por una corriente de arco dc que simula un rayo, la cual se presenta a continuación: 
$\mathrm{P}_{\text {total }}=\mathrm{P}_{\text {ele }}+\mathrm{P}_{\text {conv }}+\mathrm{P}_{\mathrm{rad}}+\mathrm{P}_{\mathrm{j}}$

En la ecuación anterior Pele es la potencia transferida por la emisión o absorción de electrones del OPGW, $P_{\text {conv }}$ es la potencia transferida por convección desde el Arco al OPGW, Prad es la potencia transferida por radiación desde el Arco al OPGW y $\mathrm{P}_{\mathrm{j}}$ es la potencia generada por el OPGW por efecto Joule. Según [3] $P_{r a d}$ y $P_{j}$ son relativamente pequeñas en comparación con $P_{\text {ele }} \mathrm{y}$ para simplificar cálculos pueden despreciarse.

Pele varía dependiendo de la polaridad del cable así:

En el caso de cátodo (cable con polaridad negativa, simula descarga eléctrica atmosférica de polaridad positiva):

$\mathrm{P}_{\text {ele }}=\left(\mathrm{V}_{c}-\mathrm{V}_{w}-\mathrm{V}_{t}\right) \times \mathrm{I}_{d c}$

En el caso de ánodo (cable con polaridad positiva, simula descarga eléctrica atmosférica de polaridad negativa):

$\mathrm{P}_{\text {ele }}=\left(\mathrm{V}_{a}+\mathrm{V}_{w}+\mathrm{V}_{t}\right) \times \mathrm{I}_{d c}$

En las ecuaciones anteriores $V_{c}$ y $V_{a}$ son las caídas de tensión en el caso de cátodo y ánodo respectivamente, $V_{w}$ es la función de trabajo del electrodo, $V_{t}$ es una caída de tensión estimado con el cociente entre la entalpía debido al arrastre de electrones y la corriente de arco ldc.

Iwata en [3] presentó los valores de las variables indicadas anteriormente los cuales se muestran en la siguiente tabla:

\begin{tabular}{|c|c|c|c|c|}
\hline Material & $\mathbf{V}_{\mathbf{c}}$ & $\mathbf{V}_{\mathbf{a}}$ & $\mathbf{V}_{\mathbf{w}}$ & $\mathbf{V}_{\mathbf{t}}$ \\
\hline Aluminio & 12,9 & 0,1 & 4,3 & 1,3 a 1,6 \\
\hline Hierro & 16,6 & 0 & 4,5 & 1,3 a 1,8 \\
\hline
\end{tabular}

Tabla 1. Caídas de tensión asociadas al calor transferido por electrones (en voltios)

*Tomado de [3].

Según [3] la potencia transferida por convección es inversamente proporcional a la longitud del gap entre el contraelectrodo y el cable. En [4] se indica que el calor transferido por Pele es de cerca del $99 \%$ y por lo tanto el calor transferido por $\mathrm{P}_{\text {conv }}$ es de cerca del $1 \%$ en el caso en que la longitud del gap entre el contraelectrodo y el cable es de $1500 \mathrm{~mm}$.

\subsection{3. ÁREA DE TRANSFERENCIA DE CALOR}

Según [3] el área de transferencia de calor de Pele y $P_{\text {conv }}$ la cual se denomina $S_{h}$, es un área tocada por el Arco y se determina con la siguiente ecuación:

$S_{\mathrm{h}}=\operatorname{MIN}\left(\frac{I_{D C}}{J_{D C}}, A_{\text {exp }}\right)$

Donde:

Idc: Corriente de arco dc, en A

Jdc: Densidad de corriente del material del cable, en $\mathrm{A} / \mathrm{mm}^{2}$

Aexp: Área expuesta del cable de guarda, en $\mathrm{mm}^{2}$

En las pruebas de arco dc se pone cinta aislante sobre el OPGW para evitar que el arco se mueva mucho con lo cual se genera un área expuesta que es el área del OPGW donde el arco podrá moverse, la longitud de OPGW que suele dejarse libre es de $40 \mathrm{~mm}$. Esta área expuesta es la que en la ecuación anterior se denomina $A_{\text {exp. }}$ Idc es la corriente de arco $D C$ de la prueba y $J_{d c}$ es la densidad de corriente del material, 60 y $33,8 \mathrm{~A} / \mathrm{mm}^{2}$ para aluminio y hierro respectivamente [3]. Debido a que el acero está compuesto principalmente de hierro se asume para los cálculos que $J_{d c}$ para el acero es el $J_{d c}$ para el hierro.

\subsection{MODELO DE CÁLCULO DESARROLLADO}

La metodología para desarrollar el modelo de cálculo se basa en el siguiente análisis:

Como se requiere comparar los resultados presentados en [3] con los del modelo desarrollado, y estos están en función del tiempo requerido para que una corriente de arco DC que simula un rayo (t\%), es necesario encontrar una ecuación que dependa de este tiempo, y t\% multiplicado por la potencia transferida por la descarga de arco DC $\left(P_{\text {ele }}\right)$ es igual a la energía necesaria para fundir un porcentaje de la sección de cable (E\%), por lo tanto, t\% es igual a E\% dividido por la potencia transferida por la descarga Pele.

Con los conceptos presentados en el numeral anterior se propone el siguiente método de cálculo del tiempo requerido para que una corriente de arco DC que simula un rayo funda un porcentaje de la sección de un cable de guarda de líneas de transmisión: 
Se debe realizar el cálculo de la energía necesaria para fundir una determinada cantidad de cable. El cálculo de la energía para cada etapa indicada anteriormente es el que se muestra a continuación:

Energía para elevar la temperatura de la cantidad de cable hasta la temperatura de fusión. Para realizar este cálculo se utiliza el concepto termodinámico de calor específico (ecuación 3.3 presentada en [5]):

$$
E 1=m \times C p \times(T f-T a)
$$

Donde:

E1: Energía para elevar la temperatura de la masa $\mathrm{m}$ de cable hasta la temperatura de fusión, en $\mathrm{J}$

$\mathrm{m}=$ masa de la cantidad de cable que se funde, en $\mathrm{kg}$

$\mathrm{Cp}=$ Calor específico del material del hilo, en $\mathrm{J} /(\mathrm{kg}$ $\mathrm{K})$

$\mathrm{Tf}=$ Temperatura de fusión del material del hilo, en $\mathrm{K}$

$\mathrm{Ta}=$ Temperatura ambiente, en $\mathrm{K}$

Energía para fundir el cable (concepto presentado en [6]):

$E 2=m \times L f$

Donde:

E2: Energía para fundir la masa $m$ de cable después de que alcanza la temperatura de fusión, en J

$\mathrm{Lf}=$ Calor latente de fusión, en $\mathrm{J} / \mathrm{kg}$

La energía total para fundir la cantidad de masa $m$ es la suma de E1 y E2. E1 y E2 no son más que la aplicación de los conceptos termodinámicos de calor específico y de calor latente de fusión, respectivamente. La cantidad de masa $m$ que se fundiría se determinará a partir de la densidad del material del cable y del volumen de cable que se funde, el cual se asume que será igual al volumen del cilindro que forman la sección transversal de un hilo del cable de longitud igual al diámetro del arco. Se aplica un factor del $30 \%$ al volumen de OPGW calculado, factor que se obtuvo de realizar una calibración del modelo realizado para ajustarse a los resultados presentados en [3]. $\mathrm{m}=$ Dens $\times$ Dar $\times A h \times 0,3$

Donde:

Dens $=$ Densidad del material del hilo, en $\mathrm{kg} / \mathrm{m}^{3}$

Dar= Diámetro del arco calculado con Sh, en m

Dar $=2 \times \sqrt{\frac{I d c}{J d c \times \pi}}$, en $\mathrm{m}$

Ah= Área o sección transversal de 1 hilo del OPGW, en $\mathrm{m}^{2}$

La energía total para fundir un porcentaje del cable, $\mathrm{E} \%$, se puede expresar mediante la siguiente ecuación:

$E \%=$ Dens $\times$ Dar $\times A h \times N h \times(C p \times(T f-T a)+$

$L f) \times 0,3$, en J $\mathrm{Ec}(8)$

Donde:

$\mathrm{Nh}=$ Número de hilos correspondientes al porcentaje de cable que se fundiría

El tiempo requerido para fundir el porcentaje del OPGW, t\%, será el tiempo durante el cual se debe transferir la potencia Pele (para longitudes de gap de $1500 \mathrm{~mm}$ se despreciará también $P_{\text {conv }}$ ) hasta alcanzar una energía igual a $\mathrm{E} \%$. Por lo tanto:

$\mathrm{t} \%=\mathrm{E} \% / \mathrm{P}_{\text {ele }}$

\section{RESULTADOS}

Para verificar los resultados obtenidos con el método desarrollado, estos se contrastarán con los resultados reportados en la Figura 8. a presentada en [3] para el tiempo requerido para fundir el $40 \%$ de un cable OPGW de hilos de ACS de conductividad del $40 \%$ IACS, de 12 hilos de $3 \mathrm{~mm}$ de diámetro (curva de color verde de la Figura 8.a en [3]), longitud de gap de $1500 \mathrm{~mm}$, con el OPGW como cátodo; por lo tanto, como se desea calcular el tiempo requerido para fundir el $40 \%$ del cable de 12 hilos, el valor para $\mathrm{Nh}$ es 4,8 (40\% de 12 hilos). Por lo tanto se debe calcular E40, con la Ec(8) así:

$E 40=$ Dens $\times$ Dar $\times A h \times 4.8 \times(C p \times(T f-T a)+$ $L f) \times 0.3$

Según lo anterior: 
Y con la $\mathrm{Ec}(9)$ se calcula t40 así:

$\mathrm{t} 40=\mathrm{E} 40 /$ Pele, en $\mathrm{s}$

A continuación se presentan valores para las variables del caso analizado:

Dens $=7874\left[\mathrm{~kg} / \mathrm{m}^{3}\right][7]$

$\mathrm{Cp}=450[\mathrm{~J} /(\mathrm{kg} \cdot \mathrm{K})][8]$

$\mathrm{Tf}=1530[\mathrm{~K}][9]$

$\mathrm{Ta}=293[\mathrm{~K}]$ (se asume $20^{\circ} \mathrm{C}$ )

$\mathrm{Lf}=293000[\mathrm{~J} / \mathrm{kg}][9]$

Dhilo $=3 \times 10^{-3}[\mathrm{~m}]$

$\mathrm{Ah}=7,69 \times 10^{-6}\left[\mathrm{~m}^{2}\right]$

$\mathrm{Jdc}=33,8 \mathrm{~A} / \mathrm{mm}^{2}$

Donde Dhilo es el diámetro de los hilos del OPGW.

En el modelo desarrollado se tuvieron las siguientes consideraciones:

El modelo desarrollado en este artículo considera que el área expuesta $\left(A_{\text {exp }}\right)$ está libre, mientras que el modelo desarrollado en [3] tiene en cuenta que en la prueba de arco el área de transferencia de energía Sh está limitado por cintas aislantes, por lo cual para corrientes mayores a 10,8 kA (corriente calculada para una longitud de cable libre de $20 \mathrm{~mm}$ y $\mathrm{A}_{\text {exp }}$ de $320 \mathrm{~mm}^{2}$ ) el modelo desarrollado no coincide con el modelo desarrollado en [3]. Sin embargo, se realizaron los cálculos para corrientes $I_{\mathrm{dc}}$ desde $1 \mathrm{kA}$ hasta $100 \mathrm{kA}$ con el fin de apreciar la diferencia de los modelos de cálculo con $A_{\text {exp }}$ libre y con $A_{\text {exp }}$ limitada.

A continuación se presentan los datos calculados de manera gráfica de $t 40$ para corrientes de arco ldc de $1 \mathrm{kA}$ hasta $100 \mathrm{kA}$ y el t40 presentado en [3], tomado como referencia:



Figura 1. Comparación de resultados del método desarrollado y del método presentado en [3].

A continuación se presenta una tabla con los datos tomados de la referencia y los datos calculados para t40 para corrientes de $l_{d c}$ desde 1 kA hasta 100 kA:

\begin{tabular}{|c|c|c|}
\hline Idc kA & $\begin{array}{c}\mathrm{t} 40 \\
\text { referencia }\end{array}$ & $\begin{array}{c}\mathrm{t} 40 \\
\text { calculado }\end{array}$ \\
\hline 1 & 0,04500 & 0,044295 \\
\hline 2 & 0,03000 & 0,031321 \\
\hline 3 & 0,02500 & 0,025574 \\
\hline 4 & 0,02100 & 0,022147 \\
\hline 5 & 0,02000 & 0,019809 \\
\hline 6 & 0,01900 & 0,018083 \\
\hline 7 & 0,01800 & 0,016742 \\
\hline 8 & 0,01700 & 0,015661 \\
\hline 9 & 0,01600 & 0,014765 \\
\hline 10 & 0,01500 & 0,014007 \\
\hline 20 & 0,00950 & 0,009905 \\
\hline 30 & 0,00650 & 0,008087 \\
\hline 40 & 0,00500 & 0,007004 \\
\hline 50 & 0,00410 & 0,006264 \\
\hline 60 & 0,00350 & 0,005718 \\
\hline 70 & 0,00302 & 0,005294 \\
\hline 80 & 0,00270 & 0,004952 \\
\hline 90 & 0,00240 & 0,004669 \\
\hline 100 & 0,00217 & 0,004429 \\
\hline
\end{tabular}

Tabla 2. Datos para t40 calculados y tomados de [3].

\section{CONCLUSIONES}

En este artículo se desarrolló un modelo de cálculo analítico del tiempo requerido para que una corriente de rayo funda un determinado porcentaje de la sección de un cable de guarda de líneas de transmisión, el cual tiene en cuenta la energía transferida al cable debido a la emisión o absorción de electrones por el cable cuando este es impactado por una descarga de arco DC que simula un rayo.

Los resultados obtenidos con el modelo desarrollado en este artículo se ajustan adecuadamente a los presentados en [3] en el rango de corrientes donde el área de transferencia de calor es menor o igual al 
área expuesta limitada con cintas aislantes con el que se realizaron los cálculos en [3].

\section{REFERENCIAS BIBLIOGRÁFICAS}

[1] Crisafulli et al. A Case Study on the Appropriate Selection of Optical Ground Wire. s.I. : IEEE, 2008. pág. 5. Power engineering conference.

[2] Alcaldía de Bogotá. PROYECTO DE ACUERDO No. 092 DE 2014: "POR MEDIO DEL CUAL SE CREA EL PROGRAMA DE PREVENCIÓN POR ALTO RIESGO DE RAYOS EN BOGOTA". [En línea] 2014. [Consultado el 01 de noviembre de 2017.] Disponible en: http://www.alcaldiabogota.gov.co/sisjur/normas/Nor ma1.jsp? $\mathrm{i}=57953$.

[3] Development of a method of calculationg the melting characteristics Characteristics of OPGW Strands Due to DC Arc Simulating Lightning Strike. Iwata et al. 3, Japón : IEEE, 2013, IEEE TRANSACTIONS ON POWER DELIVERY, Vol. 28, págs. 1314-1321.

[4] Analytical Investigation on OPGW Strands Melting due to DC Arc Discharge Simulating Lightning Strike. Iwata et al. [ed.] IEEE. Japón : IEEE, 2012. International Conference on Lightning Protection (ICLP). pág. 5.

[5] Daub et al. Química. Octava. s.I. : Pearson, 2005. pág. 72.

[6] Burbano et al. Física general. 32. s.l. : Tebar, 2003. pág. 325.

[7] Garritz et al. Química. Primera. Mexico : Pearson, 1998. pág. 90.

[8] IEEE. IEEE 80. IEEE Guide for Safety in AC Substation Grounding. s.I. : IEEE, 2000. pág. 66.

[9] N. I. Koshkin y M. G. Shirkévich. Manual de física elemental. s.I. : Mir, 1975. págs. 74-75. 